evaluating the predictions they make concerning overshadowing, the role of similarity in discrimination learning, and the effectiveness of retroactive interference. Taken together, the results that will be described lend more support to configural than elemental theories of learning.

\title{
Symposium 5: Mental representations in the brain
}

Organized by $\mathrm{C}$. Frith

\section{Symp 5/1}

MENTAL, NEURAL AND COMPUTATIONAL CORRELATES OF REPRESENTATION: HOW CLASSICAL THEORIES OF MIND LEAVE NO ROOM FOR IMPLICIT COGNITION

\section{A. Cleeremans}

Cognitive Science Research Unit, Université Libre de Bruxelles, Av. FD Roosevelt 50, Brussels B1050, Belgium

In what sense can mental representations be implicit? Does it even makesense to ask the question? Today, several constrasting perspectives on the implicit/explicit distinction have been proposed. They offer very different answers to each of three central questions about the nature of knowledge: What is conscious? What is represented? What is causally efficacious? In this talk, I review these proposals in the context of implicit learning research. For some authors, human learning is always accompanied by consciousness, whereas for others, learning may occur without concomitant changes in subjective experience. I argue that most current perspectives on this debate are rooted in the classical metaphor of mind as a symbol-processing sytem. I further argue that this perspective makes it impossible to understand implicit learning and implicit cognition, short of (1) denying the possibility of its existence (i.e., all causally efficacious representations are necessarily conscious to some extent), or (2) assuming the existence of an unconscious system ("a zombie") that is just as powerful as the conscious system, only minus consciousness. In contrast, I suggest that dynamical approaches, such as instantiated by connectionist models, make it clear how representations can be implicit in the senses of (1) being capable of influencing behavior in a way that does not require access or interpretation, and of (2) conveying information about task-relevant stimulus dimensions in a way that does not require explicit tokening of such dimensions. From this perspective thus, while awareness often accompanies learning, it is not always necessary for it to occur. 


\section{Symp 5/2 \\ MENTAL REPRESENTATION IN THE BRAIN \\ C. Frith \\ Wellcome Department of Cognitive Neurology, Institute of Neurology, University College London, UK}

Neurophysiologists frequently describe activity in neurons as representing features like colour, motion or even 'to-be-attended information in working memory'. However, these neural representations need not be associated with mental representations, that is awareness of the information represented by the neural activity. Using functional imaging techniques it is now possible to locate neural activity associated with mental representations and to contrast this with activity that is not associated with awareness. Such comparisons will be important for elucidating the neural basis of consciousness. Activity associated with perception can be dissociated from activity associated with sensory stimulation using situations in which sensory input remains constant while perception changes as with variations in attention or anomalous perceptions including hallucinations. Such studies reveal neural activity associated with awareness in inferior temporal regions concerned with the identification of objects. Evoked potentials in the EEG have been used to study the timing of brain activity associated with awareness of actions. In these studies the time of awareness is correlated with the onset of neural activity associated with precise specification of the action. In phenomena such as perceptual rivalry and pop-out there is an abrupt change in mental representation in the absence of any change in sensory input. This abrupt change is associated with transient increases in neural activity inferior temporal regions concerned with object identification and also in parietal and premotor regions concerned with object location and reaching movements. These results converge in suggesting that mental representations are associated with neural activity occurring at the level in the information processing stream at which objects and the actions appropriate to them are specified.

\section{Symp 5/3}

\section{IMPLICIT KNOWLEDGE IN DEVELOPMENT}

\section{J. Perner}

Department of Psychology, University of Salzburg, Hellbrunnerstrasse 34, A-5020 Salzburg, Austria

In several domains of cognitive development new knowledge becomes operative first implicitly (presumably inaccessible to consciousness) before it affects conscious representations. After a brief overview of different types of evidence I focus on our own research which deals with children's acquisition of mental concepts, in particular, the concept of belief as central to our folk (belief-desire) psychology. Explicit use of this concept (Wimmer \& Perner 1983) develops around the age of four years. Clements \& Perner (1994) have demonstrated the existence of a substantially earlier but implicit understanding of false belief in children aged as young as three years. Children's eye movements are monitored as they listen to a false belief story, in which the protagonist reappears either at location $\mathrm{A}$ 
(where he mistakenly thinks the desired object is) or at location B (where the object really is). Most children from 2 years and eleven months on will look to the location A when almost all children this age answer the question where the protagonist will reappear with location B. Importantly, in a control condition where the protagonist knows that the object is at $B$, none of these children look to A. The same dissociation was observed when children had to move a matto A or B to catch the protagonist coming down a slide. A majority of children who moved the mat spontaneously moved it to location $\mathrm{A}$ (where they tended to look) while children who hesitated tended to moved it to B (corresponding to their verbal answers). Since 3 year old children cannot be queried about being conscious of their response strategies, it is remarkable that correct A responses increase when the response mode is unreflective (spontaneous) action (moving mat or looking) but not when the response is delayed or verbal. This pattern of results conforms to findings from visual illusions (e.g., Bridgeman, et al., 1997) and blindsight patients (review: Rossetti, 1998), suggesting that children's knowledge underlying their correct responses is subconscious. A potential alternative interpretation is that children consciously entertain that the protagonist might appear at location A, but are uncertain about this possibility so that they do not use it for verbal responses or after deliberation (hesitating action). However, Ruffman et al. recently showed that although children are able to indicate their uncertainty by distributing poker chips 50-50 to locations $A$ and $B$ when they do not know where the protagonist will appear still put all their chips to location B even when they look to A in expectation.

\section{Symp 5/4}

\section{MENTAL REPRESENTATION IN MONKEYS}

\section{R. M. Ridley* and H. F. Baker \\ MRC Comparative Cognition Team, Dept of Experimental. Psychology, Downing St., Cambridge CB2 $3 E B, U K$}

Monkeys have been used in neuropsychological investigations because their neuroanatomy and cognitive capability have a 'surface' resemblance to that seen in humans. It is, however, important to consider the levels of mental representation at which these species operate in order to make a proper assessment of the similarities in cognitive performance between monkeys with experimentally-induced lesions and the clinical entities which these lesions are intended to model. The similarity in anatomy of the sensory apparatus and the correspondence between neurophysiological recordings in monkeys and psychophysical measures in humans suggest that primary mental representations of perception are similar in both species e.g. that when a monkey and a person look at a banana they both perceive a yellow food item. The 'yellowness' exists only in consciousness and, in this respect, monkeys and people inhabit a similar conscious domain. An interesting abnormality of consciousness encountered in clinical practice, which includes a defect in primary mental representation, is spatial hemineglect. This can be reproduced and fractionated in monkeys with experimentally-induced unilateral stroke. Secondary mental representation is necessary for recollection of events of the past, rather than merely for the alteration of 

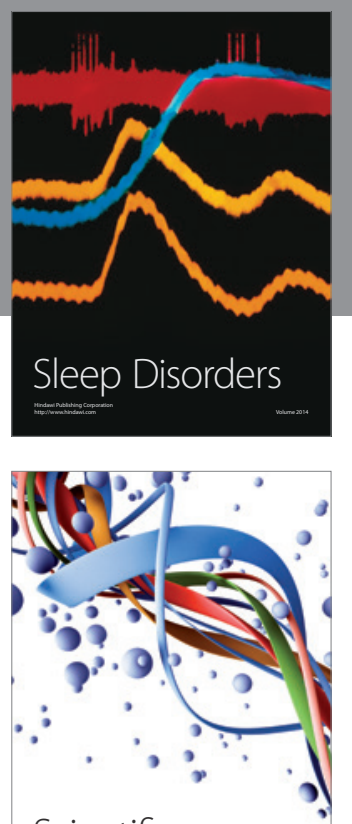

Scientifica
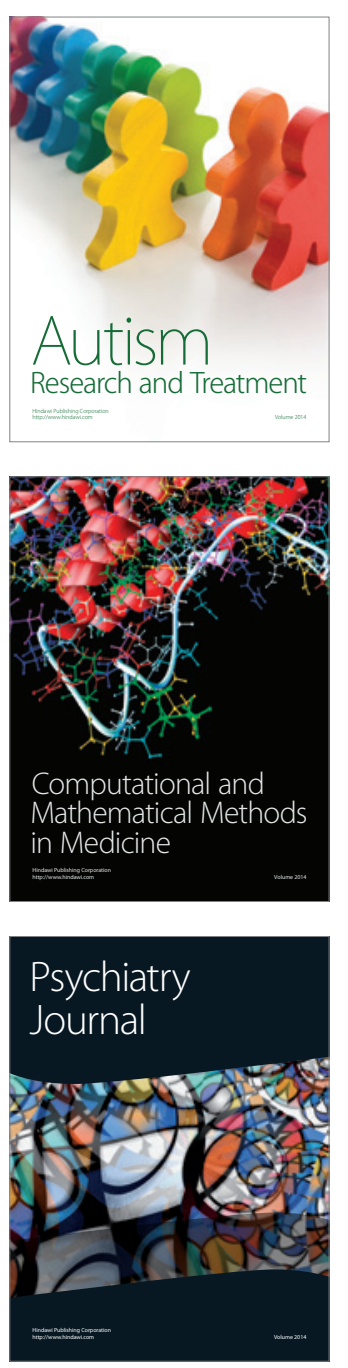
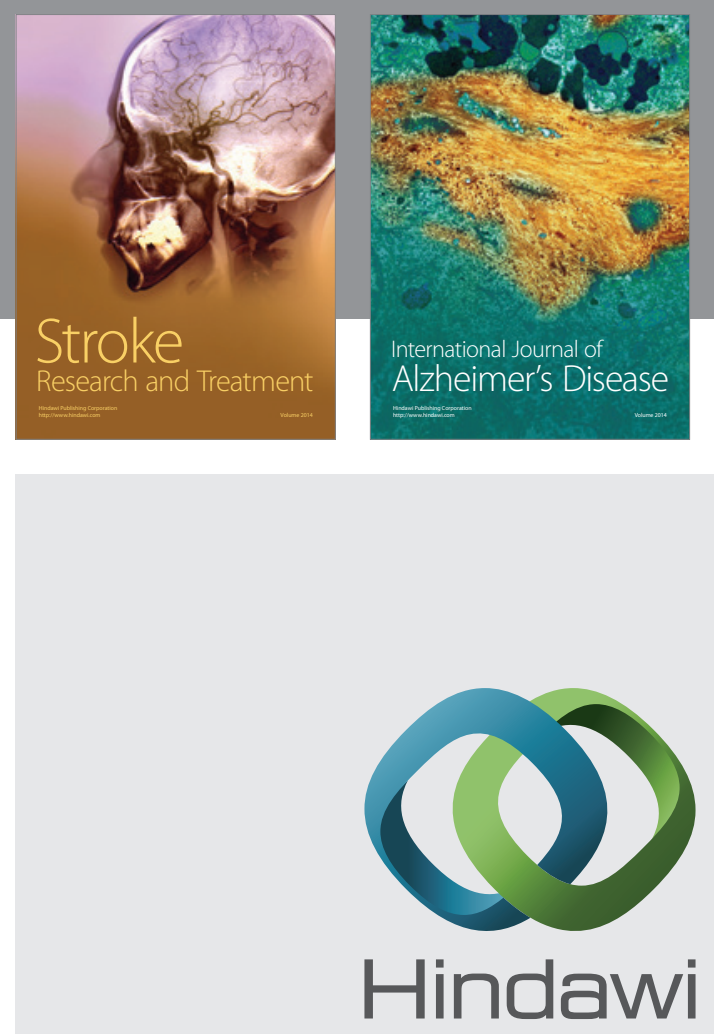

Submit your manuscripts at

http://www.hindawi.com
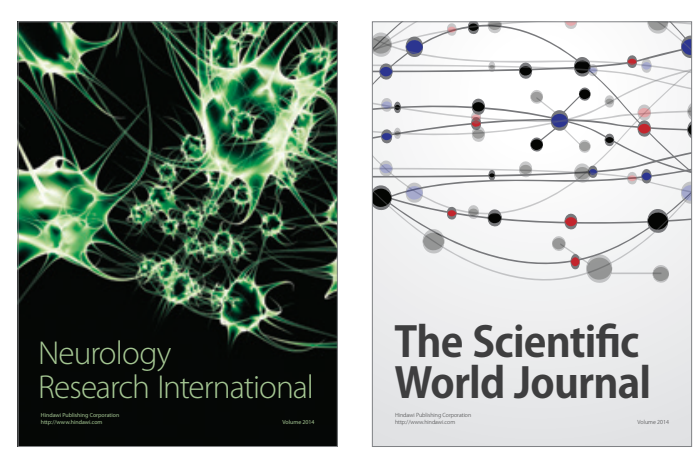

The Scientific World Journal

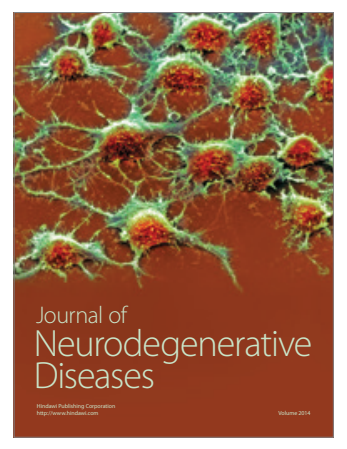

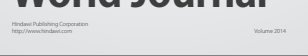

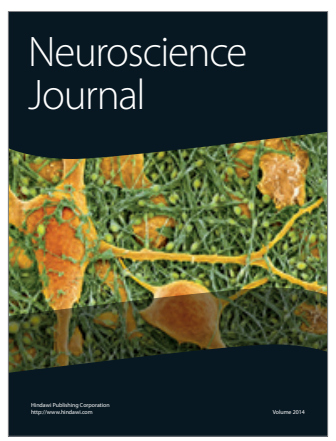

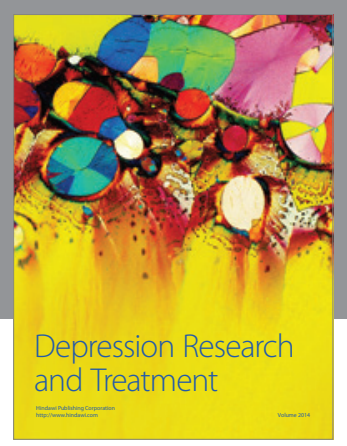
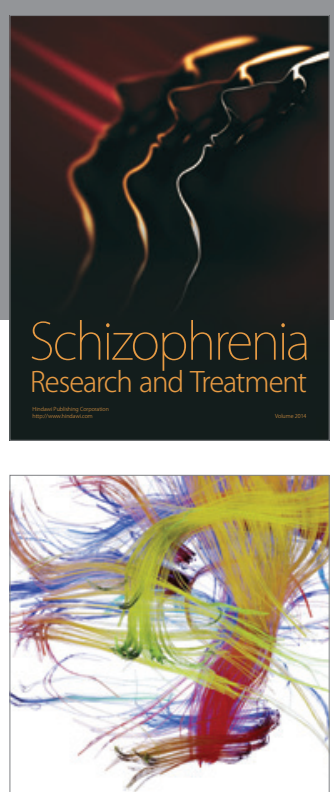

Brain Science

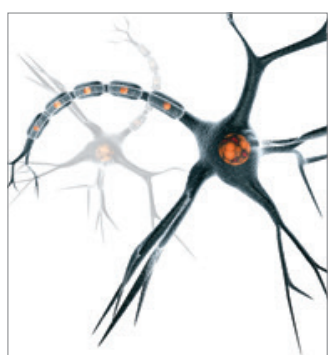

Neural Plasticity
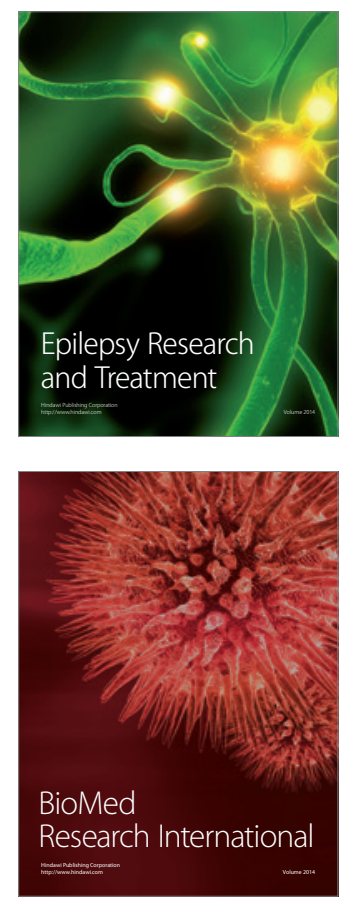

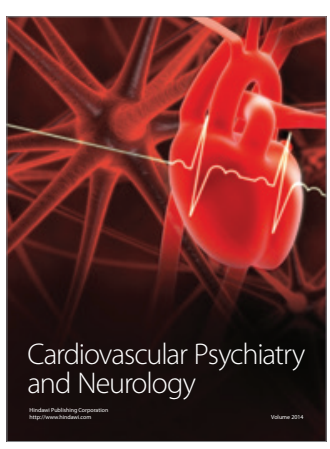

Parkinson's

Disease
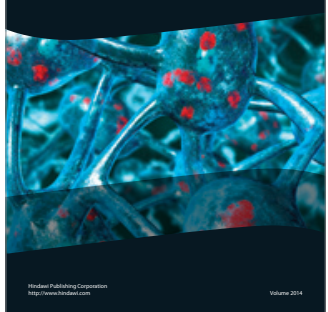